\title{
Notes sur la flore espagnole
}

(Suite et fin 1);

PAR M. MiChEL GANDOGER.

De mon voyage dans le Sud et le Centre de la Péninsule en 1906, je ne dirai que les choses nécessaires, écourté et attristé qu'il fut par la mort de ma mère ${ }^{2}$. Il avait pour but l'exploration des provinces de Cordoue, Séville, Badajoz, Ciudad Réal et Cuenca.

A Montoro, dans la province de Cordoue, je récoltai quelques centaines d'espèces, entre autres :

Fumaria spicata

- muralis

Conringia orientalis

Diplotaxis catholica

Reseda Gussonii

Lavatera cretica

Vicia amphicarpa

Tillæa muscosa

Fedia graciliflora
Carduus Reuterianus

Micropus supinus

Hedypnois tubiformis

Linaria præcox

- hirta

Ornithogalum bæticum

Lamium hybridum. Nouveau pour l'Andalousie.

\section{Province de Séville.}

La végétation primitive a presque disparu dans cette région; partout des champs de Blé et d'Oliviers. Autour de Séville, non loin de la gare, j'ai trouvé cependant :

Ranunculus trilobus

Nasturtium microphyllum

Silene apetala

Biserrula Pelecinus

Kentrophyllum bæticum
Arctotis acaulis

Pulicaria hispanica

Kalbfussia Salzmanni $F$. Sch. abondant.

Nonnea nigricans

Enfin le rarissime Triguera ambrosiacea Cav., que je voyais

1. Voir plus haut, p. 501.

2. Ma mère, née Jeanne-Marie Carriez, le 3 octobre 1826 à Pommiers (Rhône), s'intéressait beaucoup à la Floriculture et à la Botanique. Non seulement elle m'aidait dans l'arrangement de mes collections, mais souvent elle me donnait des idées très justes quand j'écrivais mes Notes de Botanique - les corrigeant au besoin - surtout quand je défendais les principes de l'école analytique si violemment attaqués par quelques-uns. - La Société botanique de France me permettra de déposer ici cet hommage de piété filiale. 
vivant pour la première fois : superbe Solanée à fleurs d'un pourpre violet et à tiges longuement diffuses.

Dans la sierra de Moron je retrouvai : Reseda Gussonii, Silene brachypetala, Thapsia decussata, Pallenis aurea, Cynoglossum clandestinum, Linaria triphylla, Orobanche speciosa var., C'hamærops humilis et une centaine d'autres.

Fixé à Utrera pendant quelques semaines j'ai rayonné, de là, dans les environs. Les bois de Pins ont heureusement conservé leur ancienne végétation; c'est surtout là qu'il faut herboriser. Voici quelques bonnes espèces :

Brassica oxyrrhina Coss.

Biscutella erigerifolia

Cistus albidus $\times$ crispus

Helianthemum punctatnm Dun.

- macrosepalum Salzm.

- intermedium

Silene clandestina Jacq. Nouveau pour la province.

- hirsuta

Erodium Salzmanni Del.

- primulaceum Welw.

Hippocrepis glauca
Ornithopus isthmocarpus Coss.

Vicia cuneata

Pimpinella villosa

Carduus bæticus $\boldsymbol{B}$. $\boldsymbol{R}$.

Hypochœris adscendens Brot.

Barkhausia heterocarpa Boiss.

Crepis gaditana Boiss.

Picridium intermedium Sch. Bip.

Scrofularia pinnatifida Brot.

Orobanche densiflora

Armeria Boissieriana.

Bromus varius.

Une sierra complètement inconnue des botanistes est celle de Gibalbin (province de Cadix). On y va d'Utrera jusqu'à la station d'El Cuervo, puis de là à pied. C'est une région intéressante; j'y récoltai près de 200 plantes, surtout :

Reseda crispata

Silene hirsuta

Hypericum bæticum

Hedysarum coronaríum

Medicago ciliaris

Hippomarathrum pterochlænum

Thapsia decusssata

Daucus muricatus var.

Krubera leptophylla

Fedia graciliflora

Echinops strigosus
Cynara Cardunculus

Carduncellus tingitanus

Anacyclus purpurascens Pers.

- radiatus

Convolvulus siculus

Cynoglossum clandestinum

Echium plantagineum $f$. albiflora Plantago Serraria

Iris alata.

Hordeum bulbosum L. Nouveau.

\section{Province de Badajoz.}

J'ai dit plus haut qu'excepté DE GoIncy et moi aucun botaniste ne paraît avoir herborisé dans cette province, du moins les 
auteurs n'en parlent pas. A Llerena j'ai trouvé d'assez bonnes choses, notamment :

Delphinium emarginatum

Ranunculus gregarius

Lupinus Termis.

Sarothamnus oxyphyllus. Nouveau.

Vicia amphicarpa

Rosa hispanica, etc.

Pimpinella villosa

Bourgæa humilis
Centaurea exarata Boiss.

Scorzonera macrocephala

Phlomis purpurea

Scrofularia pinnatifida

Ornithogalum bæticum

Ophrys apifera

Quercus Ballota, etc.

A Don Benito, capitale de la région nommée La Serena, j’ai récolté surtout : Brassica oxyrrhina, Malcolmia patula, Erodium Salzmanni, Lupinus angustifolius, Trifolium cernuum, Tillæa muscosa, Evax asterisciflora, Crepis lusitanica, Kalbfussia Salzmanni, Anchusa angustissima DC., Linaria spartea, Rumex tingitanus, R. induratus, Vulpia ambigua, Molineria lendigera et Trifolium suffocatum, nouveau pour l'Estramadure.

\section{Province de Ciudad Réal.}

Je recommande beaucoup l'herborisation de l'Atalaya près de Ciudad Réal, ville très pittoresque par ses anciens remparts rappelant ceux d'Avila et admirablement conservés Dans une journée on récoltera au moins 250 espèces. En voici les principales :

Biscutella stenophylla

Sisymbrium polyceratium

Helianthemum ægyptiacum

- retrofractum. Nouveau (n'était connu qu'à Madrid et à Grenade)

Silene tridentata

Spergularia longipes

Anthyllis cornicina

Arthrolobium durum

Lathyrus setifolius

Trifolium gemellum

Vicia calcarata

Crassula Magnolii

Fœniculum piperitum

Atractylis humilis

Anthemis incrassata

Cotula aurea
Filago pyramidata

Tolpis umbellata

Taraxacum obovatum

Convolvulus lineatus

Omphalodes linifolia

Salvia argentea

Sideritis Cavanillesii

Linaria bipunctata

Armeria allioides

Aristolochia Pistolochia

Osyris alba

Quercus rotundifolia

Asphodelus albus

Anthoxanthum aristatum

Mibora verna

Psilurus nardoides

Vulpia Michebii 
Des circonstances indépendantes de ma volonté m'empèchèrent d'explorer la province de Cuenca, complètement inconnue des botanistes, à l'exception de ce qu'en a écrit M. L. Aterido dans une excursion qu'il y fìt il y a quelques années. Voici les plantes que j’ai récoltées à Tarancon, en mai 1906 :

Adonis æstivalis

Ceratocephalus incurvus Stev.

Papaver hybridum

- Rhœas

Rœmeria hybrida

Hypecoum grandiflorum

- procumbens

- pendulum

Fumaria officinalis

Alyssum campestre

Biseutella erigerifolia

Eruca sativa

Diplotaxis virgata

Capsella Bursa-pastoris

Camelina sylvestris

Lepidium subulatum

Neslia paniculata

Matthiola tristis

Rapistrum rugosum

Sisymbrium Irio

- crassifolium

Helianthemum salicifolium

Reseda lutea $f$. hispida

- Gayana

Agrostemma Githago

Dianthus prolifer

Bufonia tenuifolia

Cerastium viscosum $f$. grandiflora

Holosteum umbellatum

Sagina procumbens

Ifalva nicæensis

Erodium cicutarium

Arthrolobium scorpioides

Astragalus hamosus

- purpureus

Medicago orbicularis

- tribuloides

- minima

- sativa

Ononis mitis

Vicia monanthos

- cuneata $f$. hirta et $f$. glabrescens

Rosa urbica

Poterium microphyllum
Torilis neglecta

Galium parisiense

Asperula arvensis

Carduus pycnocephalus

Centaurea ornata

- aspera

Cirsium castellanum

Onopordum nervosum

Xeranthemum inapertum

Anacyclus clavatus

Filago spathulata

Senecio vulgaris

Barkhausia taraxaçifolia

Podospermum subulatum

- laciniatum

Taraxacum obovatum

- Dens-leonis

Asterolinum stellatum

Lithospernum apulum

- arvense

Cynoglossum cheirifolium

Thymus Zygis

Salvia lanigera

Marrubium vulgare

Lamium amplexicaule

Phlomis Herba-venti

Linaria arvensis

Veronica opaca

- hederifolia

Plantago lanceolata

- Cynops

- albicans

Euphorbia serrata

- falcata

Mercurialis tomentosa

Brachypodium distachyon

Bromus madritensis

- tectorum

Cynodon Dactylon

Echinaria capitata

Hordeum murinum

Nardurus tenellus

Scleropoa rigida

Poa crispa 
Poa bulbosa

Vulpia ciliata.

Brachypodium phœ́nicoides $f$. hispida, très semblable et peut-être identique au B. Gandogeri, espèce portugaise que M. Hackel m’a dédiée et qui a été décrite ici, en 1910.

En somme, d'après ce que nous savons de Cuenca, sa végétation est celle du plateau de Castille. Mais il est certain que dans les sierras inexplorées de l'Est on rencontrera bien d'autres choses et que la flore doit en être assez semblable à celle de l'Aragon occidental.

Avant de rentrer en France je fis une nouvelle excursion à Aranjuez (Madrid), afin d'y récolter le Vella, Pseudocytisus L. Malgré de minutieuses recherches exécutées soit par moi-même, soit par des gens que j'avais instruits, je n'ai pu le rencontrer. A mon passage à Madrid, mon ami M. L. Aterido, auquel je suis redevable de tant de bonnes plantes, m'a dit qu'il y a une douzaine d'années il ne parvint à en trouver qu'un seul individu. Il est donc à craindre que cette espèce ne soit complètement éteinte, car elle n’était connue qu'à Aranjuez (Bourgeau, $\mathrm{n}^{\circ} 2100$ ! Cutanda! Graells! Aterido!) et dans la sierra de Maria Almeria (Bourgeau, no 1054!); mais dans cette dernière localité elle n'y a pas été retrouvée depuis 1851, époque où BouRgEaU en récolta des échantillons si malingres, si souffreteux, qu'on peut supposer que, là aussi, l'arbuste a disparu.

Je profitai de mon séjour à Aranjuez pour y récolter abondamment les rares : Clypeola eriocarpa, Iberis subvelutina, Helianthemum lineare, Frankenia Reuteri, Reseda bipinnata, Alsine conferta, Gypsophila hispanica, G. Struthium, Erodium Cavanillesii, Sedum gypsicolum, Pimpinella dichotoma, Hohenackeria polyodon, Campanula fastigiata, Linaria filifolia, Odontites hispanica, Ephedra scoparia, Colchicum triphyllum, Koleria castellana, Trisetum pumilum, Tr. Lœflingianum, et cent autres tout aussi intéressantes, Aranjuez étant une des plus riches stations botaniques de l'Espagne. 


\section{$2 \mathrm{BHL}$ Biodiversity Heritage Library}

Gandoger, Michel. 1910. "Notes sur la flore espagnole (Suite et fin)." Bulletin de la Société botanique de France 57, 564-568.

https://doi.org/10.1080/00378941.1910.10832266.

View This Item Online: https://www.biodiversitylibrary.org/item/8679

DOI: https://doi.org/10.1080/00378941.1910.10832266

Permalink: https://www.biodiversitylibrary.org/partpdf/160901

\section{Holding Institution}

Missouri Botanical Garden, Peter H. Raven Library

\section{Sponsored by}

Missouri Botanical Garden

\section{Copyright \& Reuse}

Copyright Status: Public domain. The BHL considers that this work is no longer under copyright protection.

This document was created from content at the Biodiversity Heritage Library, the world's largest open access digital library for biodiversity literature and archives. Visit BHL at https://www.biodiversitylibrary.org. 